\title{
The Appearance of Keplerian Polyhedra in an Illusory Architecture
}

\author{
Cristina Càndito \\ Andrea Quartara \\ Alessandro Meloni
}

\section{Abstract}

The illusion of ancient frescoes takes on a new form through the methods offered by digital technologies. This contribution deals with the representation of a geometric solid in the decoration of the Room of Leda in Palazzo Balbi Senarega in Genoa: this is the small stellated dodecahedron described by Johannes Kepler in 1619. An in-depth documentary and iconographic research has allowed us to prove that this is the first perspective representation of this solid which took place about thirty-five years after its discovery. The aim is to investigate and illustrate in a communicative, but not simplifying way, the connections between an example of Genoese decoration and the wider seventeenth-century milieu, which features artists, scientists, and generous patrons with their unsung brides.

Keywords

stellated polyhedra, Kepler, illusory architecture, perspective, augmented reality.

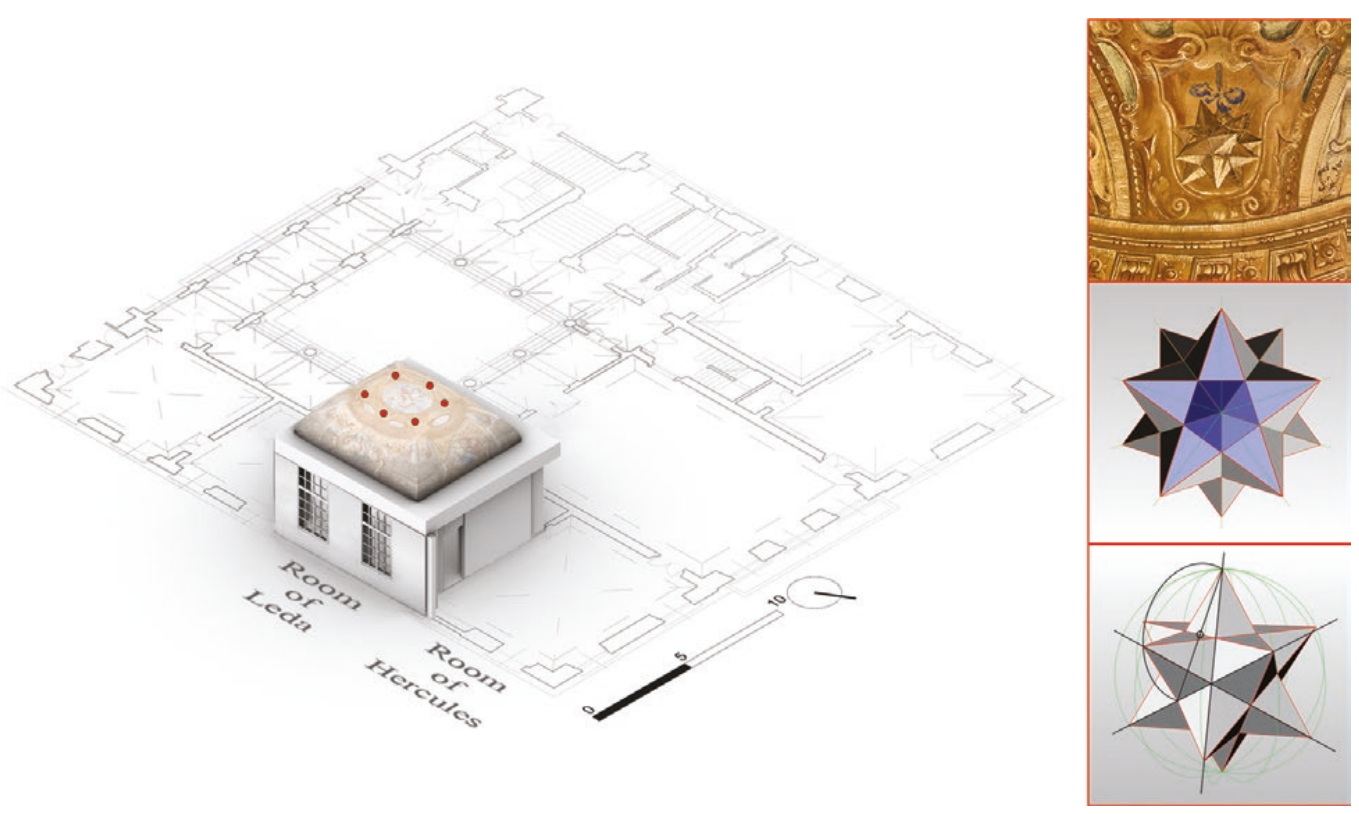




\section{Architecture and Polyhedra in the Room of Leda}

This contribution offers an updated representation of the history and meaning of some polyhedra painted in the Room of Leda, in Palazzo Balbi Senarega, in Genoa [I].The Palace, which has been part of the University of Genoa since 1972, was built by Bartolomeo Bianco around 1616 for the brothers Giacomo and Pantaleo Balbi. In 1645 it passed to Francesco Maria Balbi who wanted to enlarge the building and, starting from 1655, hired the best painters and quadraturists for the decoration of the second noble floor.

The painterValerio Castello and the quadraturist Andrea Sighizzi created the frescoes in the Room of Leda which probably belonged to Barbara Ayrolo, wife of Francesco Maria Balbi who occupied the adjacent Room of Hercules.

To identify characteristics and appropriate modes of representation, we carried out the following analysis [2]:

I. Photogrammetric surveys and nodal photography.

2. Geometric analysis of the represented perspective.

3. Application of perspective restitution procedures.

4. Material and documentary investigations on the polyhedra.

5. Modeling of illusory architecture.

6. Applications of Augmented Reality techniques.

In the Room of Leda, the painted architecture replaced the actual pavilion vault with a golden oval vault, with a large central eye in which the myth of Leda and Zeus was represented. The intent was to fully integrate various forms of art according to the Baroque style. In the present contribution, we improved our investigations about the relationships between real and illusory space, starting from the perspective restitution procedures. In the absence of geometric-proportional references, they are based on the alignment of the projective lines from a point of view $(V)$ generated by an observer in the centre of the room.

The illusory architecture of the oval vault may have been inspired by illustrious coeval architectures in Rome. The oval shape, perhaps prompted by the rectangular plan of the room, may refer to the vault of San Carlino alle Quattro Fontane by Francesco Borromini (16341644), whose shape is also echoed in the later Sant'Andrea al Quirinale ( 1658 ) by Gian Lorenzo Bernini and in other famous planimetric shapes of sixteenth and seventeenth century architectures, as the helical staircases in Palazzo del Quirinale by Ottaviano Mascarino (late sixteenth century) and in Palazzo Barberini by Borromini (1625-1633).

The profile of the illusory vault, in its complexity, could be also be compared with Guarino Guarini's vaults as the one in San Lorenzo (Turin, 1668-1687), with their oval openings and their balustrades, that in Turin is the base of the lantern and in Genoa prelude to the sky.

The representation of architecture is attributed to the quadraturist Andrea Seghizzi as well as probably the geometric elements in the Room of Leda, such as the polyhedron, repeated six times. An in-depth documentary and iconographic research on the polyhedron allowed us to prove that it was the first perspective representation of one of the solids described by Johannes Kepler in 1619: the small stellated dodecahedron, which, together with the great stellated dodecahedron, implements the small array of the five regular solids, extending them to non-convex polyhedra. Recent researches on frescoes have also provided an opportunity to obtain new insights into the relationship between art and science in a complex cultural environment of the mid-seventeenth century [3].

\section{The Reconstruction of Illusory Space}

The implemented virtual model is a geometric interpretation of the architectural scene represented in the painting of the intrados of the vaulted ceiling of the Room of Leda. Once built the photo-modelling of the vault, the geometric reconstruction of the main architectural and decorative elements took place.

As the first step, we realized a digital model of the room; in this way all the main real architectural elements (i.e. perimeter walls, doors, windows and the frame that run along the impost) configure the digital space in which the 3D photo-modelled mesh has been inset. 
Once the real space is configured, we move on to the construction of the virtual space: namely the one depicted on the vaulted ceiling. The imaginary observer - who is standing in the center of the room - individuates the point of view $(\mathrm{V})$ : all the subsequent reconstruction steps relate to this point, in order to achieve a straight geometric reconstruction. The point of view $(\mathrm{V})$ is placed at 1.7 meters (hv) from the floor level, simulating the height of a human observer, because we cannot establish a point of view through the internal perspective references. Therefore, a virtual camera is placed at this height and it is tilted zenithal upwards. In order to include the entire space of the vault in a single shot, we choose a wide-angle focal length (about $17 \mathrm{~mm}$ ). Here it is the first sensible approximation: as is known, wide-angle focal lengths deform the reality, especially in the edges of the frame. In fact, a focal length close to a $50 \mathrm{~mm}$ on a full frame camera should be used in order to obtain a photographic image resembling the unaided eye view (the so-called "normal vision"). This focal length represents the optimum value, since it impresses on the frame an image similar to the one perceived by the human eye and it is therefore able to reproduce a correct proportion of the subjects, according to all three dimensions simultaneously (height, width and depth). By accepting this optical-photographic simplifications, the following 3D modeling procedures turn in a digital model the main architectural elements painted on the golden oval vault. Namely parapet, coupled lonic columns, medallions, frame, windowed vault, polyhedra and balustrade above the central oval oculus. By observing a symmetrical arrangement of the depicted scene, two orthogonal symmetry axes ( $x$ and $y$ in fig. Ia) are defined overlying the hypothetical observer.

(a)

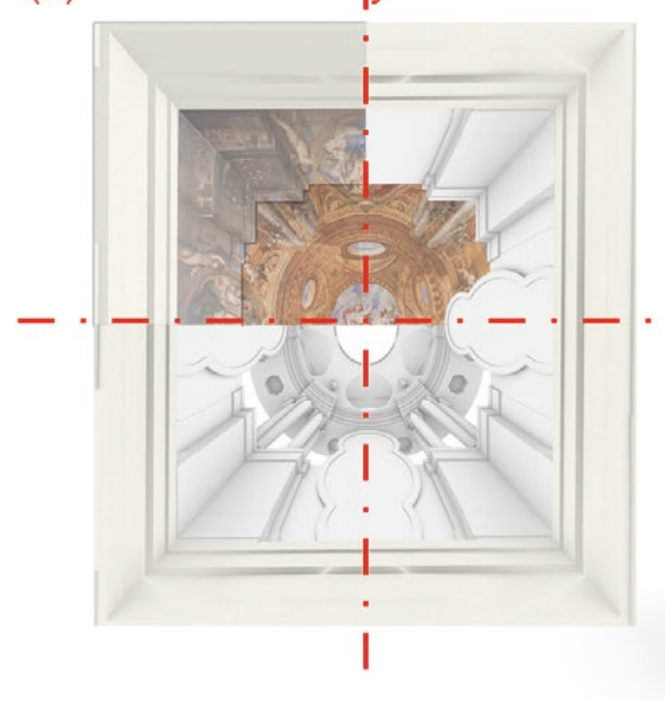

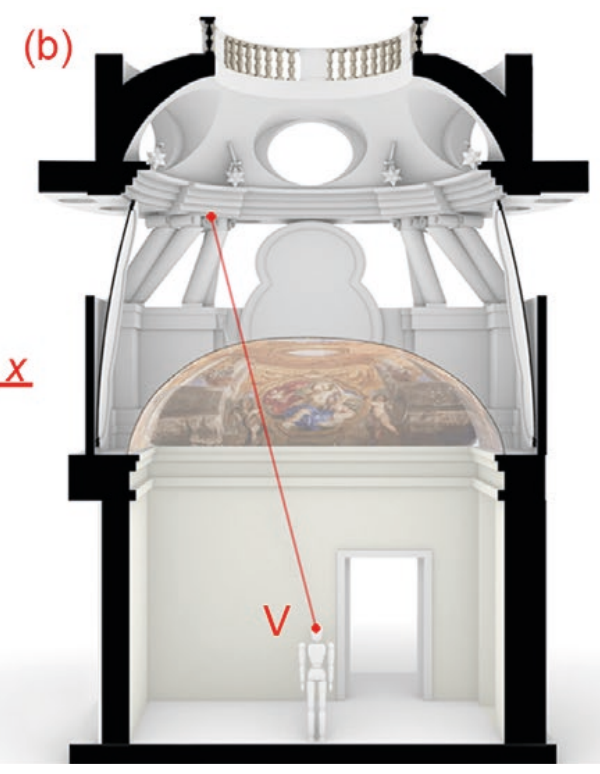

The virtual space modeling followed a workflow consisting of two main phases. The first, using perspective projection rays originating in $\mathrm{V}$, defines the heights of elements to be modelled, while the second returns the final positioning of the architectural parts. In this second step the initially modeled solids overlays with the image in rectilinear projection (photoplan) framed by the human eye positioned in $\mathrm{V}$ : by adjusting the edges contours and their alignments, the correct approximation of the architecture represented in the painting is achieved. Through the combination of perspective projection and alignment to the photo-modelled mesh texture we carefully modelled every single primary element.

As an example, the sequence applied for the construction of the parapet is exposed.

Phase I: the projection rays are thrown starting from the observer point of view (fig. Ib); the sizes of moldings (base and coping) and the ones of the parapet vertical body have been defined by means of the relative 2D geometric reconstruction in elevation.

Phase 2: the correct overlaps between the painting of the vault framed from point $V$ and its solid model are adjusted in order to obtain the best possible alignment. To achieve the 
whole parapet model, the element is then mirrored according to the $x$ and $y$ axes previously described. As a general rule a vertical arrangement of the elements was assumed. However, we observed an incompatibility in the position of the bases of the columns compared to their capitals which are not vertically aligned. This misalignment was verified through the simulation of an ideal configuration; in the vertical alignment, in fact, the capital would be positioned in the annular extension of the frame of the vault. With the described structural issue, we also recognized a contradiction in the figurative superimposition of the frescoes. These were the reason why we adopted the choice of an oblique arrangement of the columns nevertheless recognizing its incompatibility with an ideal architecture. Furthermore, again to respect the result of the superimposition, an irregular shape of the oculi has been assumed which allows to visualize the cusp of the lunettes.

The modeling of the virtual architecture represented on the vault of the Room of Leda therefore achieves a good approximation by returning a navigable digital environment where the user can observe both the architectural and the decorative elements. The six stellated dodecahedrons hanging with ribbons on the surface of the windowed vault are positioned in the six pendentives symmetrically.

\section{Augmented Reality Applications}

The digital model of the architectural space was implemented in its augmented reality version using Unity software (Unity Technologies) together with Vuforia plug-in, thus generating the application to be installed on a mobile device to make visible the reference image and the three-dimensional model associated with it (fig. 2a).

The available augmented reality technology definitely oversteps the two-dimensional representation's limits, providing a set of tools that allow overtaking the simple visualization by offering an immersive experience. AR tools expand the spatial investigation possibilities and they are at once resources for communicating the knowledge of the place which one comes in contact with. Neil Spiller, for example, used augmented reality applications in an unusually suggestive way within the Walled Garden for Lebbeus project [Spiller 20I4]. In his personal and poetic eulogy to Lebbeus Woods, he overlapped virtuality on the image in order to inspire emotions: in this way he introduced within representation the environmental factors (e.g. weather phenomena and phantasmagorical presences) affecting the spatial reading and that permit to investigate in more detail its representation. Augmented or mixed reality technologies can be used for dissemination purposes to take advantage of spatial

Fig. 2. Elevated and stellated dodecahedra, the red line highlights the continuity or discontinuity of the sides. (a) View of the small Keplerian stellated dodecahedron in the Room of Leda through Augmented Reality (b) Kepler 1619 Reality. (b) J. Kepler, 1619 (c) P. Uccello, 1425. (d) L. Pacioli, I 509. (e) D.
Barbaro, I 569.

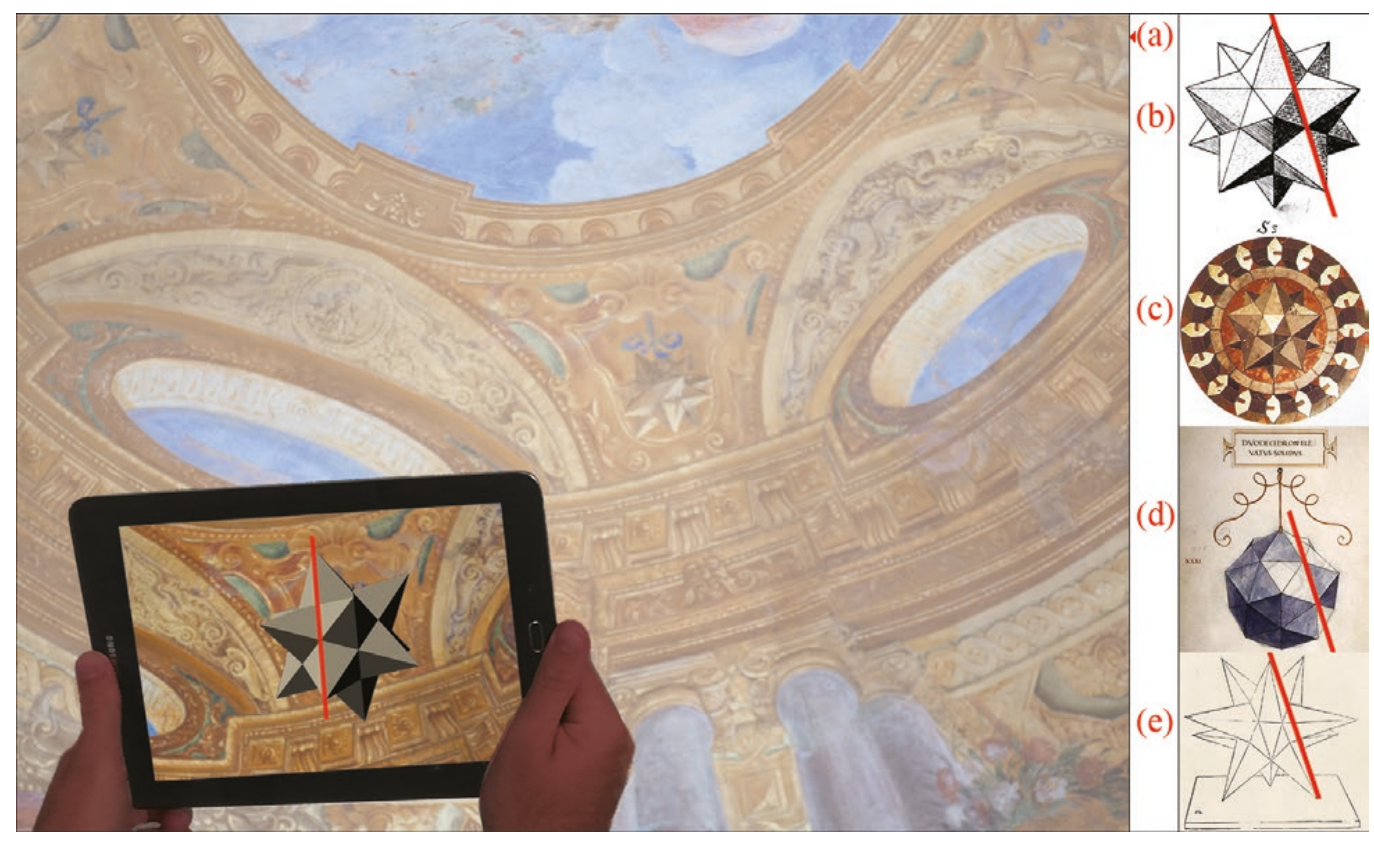


analysis features and streamlining the interaction process between the architectural environment and the viewer, while making it intuitive and direct. The application of this systems allowed us to highlight differences between the unaided eye view of the Room of Leda frescoes and their virtual reconstruction. Moreover, it was possible to focus on the understanding of the small stellated dodecahedron, which turn out to be the first perspective representation of this Keplerian solid, as claimed previously. It may be interesting to show the peculiarity of this prodigious solid by exploiting the highly communicative and immersive AR experience. In fact, the small Keplerian stellated dodecahedron is distinguished from the common elevated dodecahedron thanks to the continuity between the sides of the pentagons of the original dodecahedron, with the edges of the pyramids of the contiguous stars (fig. 2b): a property that can be better understood through virtual model that appears in our devices enhanced by perceptual information. The realtime interaction with the 3D solid emphasizes its peculiar geometric properties. Thanks to this observation, the iconographic meanings of this innovative scientific presence can be illustrated in a simple way in a seventeenth-century fresco of a lady's bedroom, whose beauty is honored thanks to the golden ratio harmonies of the geometric solid [4]. The comparison with its illustrious predecessors is of interest too. So, we can show the orthogonal representation of the solid appeared in a mosaic of the basilica of San Marco, traditionally attributed to Paolo Uccello (around I425) (fig. 2c). We can also cite the illustration by Leonardo da Vinci, contained in De divina proporzione by Luca Pacioli ( 1509 ) which represented a generic elevated dodecahedron with reduced projections and the consequent lack of continuity described (fig. 2d), which was also found in the perspective illustration by Daniele Barbaro (The practice of perspective, 1569) which described an elevated dodecahedron with much taller pyramids (fig. 2e).

Room of Leda and its features (spatial, geometric, artistic and historical) should be perceived by the widest possible audience. The use of augmented reality allows us to make them also accessible to people with disabilities. The use of an easy-to-handle device is preferable by people with mobility impaired. The possibility of using transcriptions and audio descriptions regarding the main characteristics can help to enhance accessibility for people with hearing and visual disabilities. It is therefore an intervention that implements inclusivity, addressing different age groups and people with cognitive disabilities too. AR technologies experience is seamlessly interwoven with the physical world such that it is perceived as an immersive aspect of the real environment full extent and not competing the frescoes, helping in clarifying some artifices of the past.

\section{Notes}

[I] The paper was conceived and elaborated as a team-work: the paragraph Architecture and polyhedra in the Room of Leda was written by C. Càndito, The reconstruction of illusory space by A. Quartara, and Augmented Reality Applications by A. Meloni.

[2] Analysis from I to 4 were realized by Cristina Càndito, with the collaboration of llenio Celoria for the point I; operation 5 by Andrea Quartara and 6 by Alessandro. Meloni.

[3] For the identification of the polyhedron and the artistic and scientific influences on Seghizzi's work, cf. Càndito, Celoria [in press].

[4] For meanings related to golden ratio perfection and hypotheses about their implications, cf. Càndito, Celoria [in press].

\section{References}

Kepler Johannes (1619). Harmonices Mundi. Linz: Johann Planck.

Càndito Cristina, Celoria Ilenio (in press). Sources and features of the small stellated dodecahedra painted in Genoa. In Proceedings of Geometrias' 2019 - Polyhedra and beyond (APROGED). Porto 2019.

Magnani Lauro (2008). Il ciclo di Valerio Castello nel palazzo di Francesco Maria Balbi. In Cataldi Gallo Marzia, Leoncini Luca, Manzitti Camillo, Sanguineti Daniele (eds.). Valerio Castello I624- I659. Genio Moderno. Milano: Skira, pp. 65-77.

Spiller Neil (20 I4). Detailing the Walled Garden for Lebbeus. In Architectural Design, 84 (4), pp. I I 8- I 27.

\section{Authors}

Cristina Càndito, Dept. of Architecture and Design, University of Genoa, cristina.candito@unige.it

Andrea Quartara, Dept. of Architecture and Design, University of Genoa, andreaquartara@gmail.com

Alessandro Meloni, Dept. of Architecture and Design, University of Genoa, alessandro.meloni@edu.unige.it 
